Electronic Supporting Material on the publication entitled

\title{
In silico rational design and virtual screening of bioactive peptides based on QSAR modeling
}

\section{Mehri Mahmoodi-Reihani a, Fatemeh Abbasitabar b,*, Vahid Zare- Shahabadi a}

a Department of Chemistry, Mahshahr Branch, Islamic Azad University, Mahshahr, Iran

b Department of Chemistry, Marvdasht Branch, Islamic Azad University, Marvdasht, Iran 


\section{Table of Contents}

Table S1. AA indices used in the construction of the ACE QSAR model. .....................................................

Table S2. Calculated numerical descriptive matrix (NDM) for ACE data set together with the experimental and predicted biological activities.

Table S3. Physicochemical indices of amino acids used to calculate numerical descriptive vector for bitter peptide.

Table S4. Calculated NDM for BBT data set together with the experimental and predicted biological activities.

Table S5. Statistical analysis of the selected QSAR model for BTT data set. ..........................................

Table S6. QSAR models for HLA data set obtained using different sets of the AA indices.........................8 
Table S1. AA indices used in the construction of the ACE QSAR model.

\begin{tabular}{lrr}
\hline AA index & min & \multicolumn{1}{c}{ max } \\
\hline Signal sequence helical potential (Argos et al., 1982) & 0.05 & 3.23 \\
Short and medium range non-bonded energy per residue (Oobatake-Ooi, 1977) & -14.42 & -5.46 \\
Weights for coil at the window position of -4 (Qian-Sejnowski, 1988) & -0.43 & 0.33 \\
Weights for coil at the window position of 1 (Qian-Sejnowski, 1988) & -0.99 & 2.27 \\
Information measure for extended without H-bond (Robson-Suzuki, 1976) & -3.70 & 7.40 \\
Information measure for N-terminal turn (Robson-Suzuki, 1976) & -4.30 & 6.50 \\
Alpha helix propensity of position 44 in T4 lysozyme (Blaber et al., 1993) & -2.50 & 0.96 \\
Hydrophobicity coefficient in RP-HPLC, C18 with 0.1\% TFA/MeCN/H $\mathrm{H}_{2} \mathrm{O}$ (Wilce etal. & -2.24 & 4.80 \\
1995) & & \\
\hline
\end{tabular}


Table S2. Calculated numerical descriptive matrix (NDM) for ACE data set together with the experimental and predicted biological activities.

\begin{tabular}{|c|c|c|c|c|c|c|}
\hline & Sequence & $\mathrm{Nu}$ & al descri & vector & Experimental & Predicted \\
\hline Training & VVV & -16.059 & -17.474 & -16.059 & 1.63 & 1.951 \\
\hline Training & RPG & -15.869 & -18.640 & -10.460 & 3.09 & 2.913 \\
\hline Training & GRP & -10.709 & -16.374 & -18.336 & 0.48 & 1.191 \\
\hline Training & LLL & -19.907 & -21.660 & -19.907 & 1.35 & 1.561 \\
\hline Training & GLG & -12.276 & -18.156 & -12.276 & 2.45 & 2.354 \\
\hline Training & LGL & -18.060 & -14.667 & -18.060 & 1.52 & 1.728 \\
\hline Training & FGG & -18.470 & -12.909 & -10.830 & 2.79 & 3.002 \\
\hline Training & GFG & -12.157 & -19.112 & -12.157 & 2.53 & 2.370 \\
\hline Training & GGF & -10.830 & -12.909 & -18.470 & 1.11 & 1.162 \\
\hline Training & FFG & -20.986 & -21.518 & -12.740 & 2.71 & 2.875 \\
\hline Training & FGF & -19.376 & -14.717 & -19.376 & 1.29 & 1.589 \\
\hline Training & GFF & -12.740 & -21.518 & -20.986 & 1.02 & 0.889 \\
\hline Training & GGG & -10.452 & -11.373 & -10.452 & 2.61 & 2.520 \\
\hline Training & GYG & -11.467 & -18.638 & -11.467 & 2.33 & 2.441 \\
\hline Training & GGY & -10.405 & -12.211 & -18.099 & 1.35 & 1.195 \\
\hline Training & YGY & -19.194 & -13.875 & -19.194 & 1.82 & 1.605 \\
\hline Training & GYY & -11.956 & -21.384 & -21.008 & 1.07 & 0.832 \\
\hline Training & YYY & -21.965 & -23.900 & -21.965 & 1.54 & 1.352 \\
\hline Training & FIV & -20.314 & -18.730 & -16.134 & 2.04 & 2.231 \\
\hline Training & FPP & -20.214 & -22.188 & -20.771 & 1.50 & 1.434 \\
\hline Training & FPK & -20.046 & -20.149 & -15.551 & 2.45 & 2.319 \\
\hline Training & PFP & -20.270 & -21.894 & -20.270 & 1.74 & 1.523 \\
\hline Training & RRR & -16.445 & -17.894 & -16.445 & 1.77 & 1.912 \\
\hline Training & PPP & -21.211 & -23.080 & -21.211 & 1.86 & 1.429 \\
\hline Training & FFF & -21.809 & -23.731 & -21.809 & 1.20 & 1.368 \\
\hline Training & RGP & -14.714 & -11.989 & -17.310 & 1.73 & 1.622 \\
\hline Training & PGR & -17.310 & -11.989 & -14.714 & 2.67 & 2.247 \\
\hline Training & GGV & -10.390 & -11.634 & -13.873 & 1.99 & 1.924 \\
\hline Training & GVV & -10.928 & -16.035 & -15.508 & 1.82 & 1.694 \\
\hline Training & PPG & -20.098 & -20.235 & -10.251 & 3.18 & 3.241 \\
\hline Training & PGG & -16.635 & -10.795 & -9.652 & 3.14 & 3.074 \\
\hline Training & PGP & -18.073 & -11.965 & -18.073 & 1.82 & 1.716 \\
\hline Training & GPG & -10.021 & -17.042 & -10.021 & 2.65 & 2.588 \\
\hline Training & GGP & -9.652 & -10.795 & -16.635 & 1.28 & 1.392 \\
\hline Training & PGI & -17.183 & -11.993 & -14.779 & 2.23 & 2.227 \\
\hline Training & KPK & -15.462 & -18.690 & -15.462 & 2.63 & 2.019 \\
\hline Training & ADA & -12.482 & -15.568 & -12.482 & 2.17 & 2.322 \\
\hline Training & GEG & -11.098 & -15.803 & -11.098 & 2.28 & 2.469 \\
\hline Training & LEL & -18.749 & -18.799 & -18.749 & 1.19 & 1.672 \\
\hline Training & RGP & -14.714 & -11.989 & -17.310 & 1.73 & 1.622 \\
\hline Training & PIP & -19.358 & -17.903 & -19.358 & 1.69 & 1.604 \\
\hline Training & FPF & -20.665 & -21.651 & -20.665 & 1.32 & 1.481 \\
\hline Training & KPF & -15.551 & -20.149 & -20.046 & 1.51 & 1.237 \\
\hline Training & VYP & -16.493 & -21.952 & -20.309 & 0.82 & 1.262 \\
\hline Training & YPF & -21.324 & -22.213 & -20.575 & 1.60 & 1.543 \\
\hline Test & LGG & -17.406 & -13.060 & -11.014 & 2.49 & 2.899 \\
\hline Test & GGL & -11.014 & -13.060 & -17.406 & 1.63 & 1.359 \\
\hline Test & LLG & -19.297 & -19.979 & -12.822 & 2.33 & 2.741 \\
\hline Test & GLL & -12.822 & -19.979 & -19.297 & 1.47 & 1.181 \\
\hline Test & YGG & -18.099 & -12.211 & -10.405 & 3.07 & 3.048 \\
\hline Test & YYG & -21.008 & -21.384 & -11.956 & 2.79 & 3.012 \\
\hline Test & LDL & -18.636 & -18.192 & -18.636 & 1.42 & 1.681 \\
\hline Test & VIF & -16.134 & -18.730 & -20.314 & 0.78 & 1.224 \\
\hline Test & RPF & -16.590 & -20.826 & -20.176 & 1.59 & 1.287 \\
\hline Test & PPF & -20.771 & -22.188 & -20.214 & 1.68 & 1.568 \\
\hline
\end{tabular}


Table S3. Physicochemical indices of amino acids used to calculate numerical descriptive vector for bitter peptide

\begin{tabular}{lrr}
\hline AA index & Min & \multicolumn{1}{c}{ Max } \\
\hline STERIMOL maximum width of the side chain (Fauchere et al., 1988) & 1.00 & 6.72 \\
Retention coefficient in HPLC, pH 2.1 (Meek, 1980) & -7.50 & 18.10 \\
Optimized side chain interaction parameter (Oobatake et al., 1985) & -0.78 & 10.68 \\
Surrounding hydrophobicity in alpha-helix (Ponnuswamy et al., 1980) & 10.98 & 15.36 \\
Average relative fractional occurrence in AL(i-1) (Rackovky-Scheraga, 1982) & 0.00 & 6.49 \\
RF rank (Zimmerman et al., 1968) & 2.80 & 18.80 \\
Surface composition of amino acids in extracellular proteins of mesophiles (percent) & 0.42 & 9.67 \\
(Fukuchi-Nishikawa, 2001) & & \\
Diameter (maximum eccentricity) (Karkbara-Knisley, 2016) & 0.00 & 14.00 \\
\hline
\end{tabular}


Table S4. Calculated NDM for BBT data set together with the experimental and predicted biological activities.

\begin{tabular}{|c|c|c|c|c|c|}
\hline & Sequence & & & Experimental & Predicted \\
\hline Training & GV & -1.456 & -1.468 & 1.13 & 1.31 \\
\hline Training & GL & -1.536 & -1.872 & 1.68 & 1.68 \\
\hline Training & GI & -1.493 & -1.650 & 1.7 & 1.68 \\
\hline Training & GP & -1.516 & -1.706 & 1.35 & 1.47 \\
\hline Training & GF & -1.572 & -2.099 & 1.8 & 2.01 \\
\hline Training & GW & -1.600 & -2.223 & 1.89 & 1.97 \\
\hline Training & $\mathrm{AV}$ & -1.461 & -1.473 & 1.16 & 1.17 \\
\hline Training & AL & -1.561 & -1.890 & 1.7 & 1.58 \\
\hline Training & $\mathrm{AF}$ & -1.606 & -2.120 & 1.72 & 1.91 \\
\hline Training & VG & -1.468 & -1.456 & 1.19 & 1.30 \\
\hline Training & VA & -1.473 & -1.461 & 1.16 & 1.18 \\
\hline Training & VV & -1.481 & -1.481 & 1.71 & 1.39 \\
\hline Training & VL & -1.602 & -1.912 & 2 & 1.86 \\
\hline Training & LG & -1.872 & -1.536 & 1.72 & 1.72 \\
\hline Training & LA & -1.890 & -1.561 & 1.72 & 1.65 \\
\hline Training & LL & -2.085 & -2.085 & 2.35 & 2.42 \\
\hline Training & LF & -2.164 & -2.330 & 2.75 & 2.81 \\
\hline Training & LY & -1.951 & -1.704 & 2.46 & 2.38 \\
\hline Training & IG & -1.650 & -1.493 & 1.68 & 1.73 \\
\hline Training & IA & -1.663 & -1.508 & 1.68 & 1.67 \\
\hline Training & IV & -1.681 & -1.540 & 2.05 & 1.93 \\
\hline Training & II & -1.759 & -1.759 & 2.26 & 2.50 \\
\hline Training & IP & -1.775 & -1.808 & 2.4 & 2.11 \\
\hline Training & IW & -1.947 & -2.367 & 3.05 & 2.93 \\
\hline Training & ID & -1.489 & -1.547 & 1.37 & 1.28 \\
\hline Training & IQ & -1.444 & -1.725 & 1.49 & 1.57 \\
\hline Training & IE & -1.436 & -1.782 & 1.37 & 1.36 \\
\hline Training & IK & -1.351 & -2.187 & 1.65 & 1.65 \\
\hline Training & IS & -1.555 & -1.439 & 1.49 & 1.46 \\
\hline Training & IT & -1.513 & -1.455 & 1.49 & 1.82 \\
\hline Training & PA & -1.718 & -1.531 & 1.32 & 1.34 \\
\hline Training & PL & -1.882 & -2.013 & 2.22 & 2.05 \\
\hline Training & PI & -1.808 & -1.775 & 2.33 & 2.07 \\
\hline Training & FG & -2.099 & -1.572 & 1.77 & 2.10 \\
\hline Training & FL & -2.330 & -2.164 & 2.87 & 2.85 \\
\hline Training & FP & -2.249 & -1.948 & 2.7 & 2.51 \\
\hline Training & $\mathrm{FF}$ & -2.415 & -2.415 & 3.1 & 3.27 \\
\hline Training & WW & -2.586 & -2.586 & 3.6 & 3.50 \\
\hline Training & YL & -1.704 & -1.951 & 2.4 & 2.38 \\
\hline Training & SL & -1.424 & -1.756 & 1.49 & 1.39 \\
\hline Test & GY & -1.473 & -1.545 & 1.77 & 1.65 \\
\hline Test & LW & -2.206 & -2.457 & 3.4 & 2.87 \\
\hline Test & IL & -1.837 & -2.000 & 2.26 & 2.46 \\
\hline Test & IN & -1.489 & -1.547 & 1.49 & 1.55 \\
\hline Test & PY & -1.767 & -1.654 & 1.8 & 2.02 \\
\hline Test & PF & -1.948 & -2.249 & 2.8 & 2.44 \\
\hline Test & FY & -2.187 & -1.767 & 3.13 & 2.85 \\
\hline Test & WE & -1.885 & -1.526 & 1.56 & 1.79 \\
\hline
\end{tabular}


Table S5. Statistical analysis of the selected QSAR model for BTT data set.

\begin{tabular}{clll}
\hline Regression coefficient & SE & t-value & $\boldsymbol{P}$-value \\
\hline 1.92 & 0.024 & 78.60 & $8.91 \times 10^{-43}$ \\
-0.33 & 0.031 & -10.74 & $6.26 \times 10^{-13}$ \\
-0.32 & 0.031 & -10.40 & $1.57 \times 10^{-12}$ \\
\hline
\end{tabular}


Table S6. QSAR models for HLA data set obtained using different sets of the AA indices.

\begin{tabular}{clllllll}
\hline Number of used AA indices & $\boldsymbol{R}_{\text {training }}^{2}$ & $\boldsymbol{R}_{\text {test }}^{2}$ & $\boldsymbol{q}_{\text {loo }}^{2}$ & $\boldsymbol{q}_{\text {lmo }}^{2}$ & $\boldsymbol{R S M} \boldsymbol{E}_{\text {training }}$ & $\boldsymbol{R S M}_{\boldsymbol{\text { test }}}$ & $\boldsymbol{R}_{\boldsymbol{M P} \boldsymbol{P}^{\mathrm{a}}}^{2}$ \\
\hline 2 & 0.504 & 0.478 & 0.415 & 0.416 & 0.690 & 0.679 & 0.034 \\
3 & 0.518 & 0.505 & 0.397 & 0.434 & 0.680 & 0.638 & 0.030 \\
4 & 0.511 & 0.507 & 0.392 & 0.416 & 0.685 & 0.661 & 0.063 \\
5 & 0.517 & 0.533 & 0.377 & 0.435 & 0.681 & 0.627 & 0.020 \\
$\mathbf{6}$ & $\mathbf{0 . 5 4 0}$ & $\mathbf{0 . 5 3 5}$ & $\mathbf{0 . 4 0 7}$ & $\mathbf{0 . 4 6 1}$ & $\mathbf{0 . 6 6 4}$ & $\mathbf{0 . 6 2 3}$ & $\mathbf{0 . 0 4 8}$ \\
7 & 0.527 & 0.530 & 0.396 & 0.450 & 0.673 & 0.627 & 0.055 \\
8 & 0.541 & 0.535 & 0.406 & 0.456 & 0.663 & 0.625 & 0.058 \\
9 & 0.549 & 0.534 & 0.397 & 0.460 & 0.657 & 0.623 & 0.041 \\
10 & 0.540 & 0.537 & 0.399 & 0.454 & 0.664 & 0.622 & 0.032 \\
11 & 0.540 & 0.535 & 0.395 & 0.456 & 0.664 & 0.629 & 0.037 \\
12 & 0.543 & 0.536 & 0.413 & 0.466 & 0.662 & 0.625 & 0.061 \\
13 & 0.514 & 0.521 & 0.373 & 0.413 & 0.683 & 0.652 & 0.054 \\
14 & 0.524 & 0.534 & 0.386 & 0.423 & 0.676 & 0.645 & 0.059 \\
15 & 0.537 & 0.531 & 0.427 & 0.453 & 0.666 & 0.647 & 0.026 \\
16 & 0.522 & 0.519 & 0.390 & 0.426 & 0.677 & 0.651 & 0.030 \\
17 & 0.521 & 0.522 & 0.387 & 0.422 & 0.678 & 0.654 & 0.029 \\
18 & 0.522 & 0.520 & 0.387 & 0.423 & 0.677 & 0.654 & 0.057 \\
19 & 0.533 & 0.511 & 0.411 & 0.445 & 0.669 & 0.659 & 0.045 \\
20 & 0.490 & 0.517 & 0.331 & 0.361 & 0.699 & 0.642 & 0.047 \\
\hline
\end{tabular}

